\title{
Manejo quirúrgico de la marcha por torsión tibial interna en niños
}

\author{
Surgical management of gait by internal tibial torsion in children
}

\author{
Oliveros-Rivero JA, ${ }^{*}$ Mendoza-Saldarreaga LG,${ }^{*}$ Forestieri-Flores M, ${ }^{\ddagger}$ Quiñonez-León J, ${ }^{\S}$ Melgar-Celleri J
}

Hospital de Niños «Dr. Roberto Gilbert Elizalde», Guayaquil, Ecuador.

RESUMEN. Introducción: Identificar la evolución en la marcha de los pacientes que presentaron torsión tibial interna y se les trató con corrección quirúrgica, realizándoles osteotomía tibial desrotadora. Material y métodos: Estudio retrospectivo, se incluyeron pacientes con diagnóstico de torsión tibial interna causante de marcha anormal, en quienes fracasó el tratamiento conservador, entre Enero de 2016 y Enero de 2019. Resultados: Se trataron 12 pacientes con rango de edad de 4.3-17.8 años, de los cuales nueve fueron femeninos $(75 \%)$ y tres masculinos $(25 \%)$; el rango de peso fue 12.36-59.30 kg; entre las patologías asociadas se encontraron mielomeningocele en tres pacientes (25\%), parálisis cerebral infantil en dos pacientes (16.6\%); por otra parte, la estancia hospitalaria postoperatoria de seis pacientes $(50 \%)$ fue de un día, cinco pacientes $(41.67 \%)$ permanecieron dos, y sólo un paciente tres días $(8.33 \%)$; tres pacientes $(25 \%)$ presentaron complicaciones menores y un paciente $(8.33 \%)$ complicaciones mayores; la evolución de la marcha fue buena en 11 pacientes $(91.67 \%)$ y regular en un paciente $(8.33 \%)$. Conclusión: Es la primera serie comunicada en el Ecuador en la que se hace referencia al uso de osteotomía desrotadora de la tibia en el área supramaleolar combinada con la fijación de clavo de Kirschner, corrigiendo la torsión tibial interna en niños en los que fracasó el tratamiento conservador, resultando un procedimiento seguro y con desenlaces favorables en la evolución de la marcha, incluyendo pacientes con enfermedad neuromuscular y menores de cinco años.

Palabras clave: Marcha, tratamiento, torsión tibial, osteotomía, evolución.
ABSTRACT. Introduction: Identify the progress of patients who had internal tibial torsion and were treated with surgical correction, performing derotating tibial osteotomy. Material and methods: Retrospective study included patients diagnosed with abnormal gait-causing internal tibial torsion in which conservative treatment failed, between January/2016 and January/2019. Results: 12 patients with an age range of 4.3-17.8 years of whom nine patients (75\%) were female and three (25\%) male; the weight range was $12.36-59.30 \mathrm{~kg}$; among the associated pathologies were found myelomeningocele three patients $(25 \%)$, cerebral palsy two patients $(16.6 \%)$; the postoperative hospital stay of six patients $(50 \%)$ one day, five patients $(41.67 \%)$ stayed two days, and three days only one patient $(8.33 \%)$; three patients (25\%) minor complications and one patient (8.33\%) major complications; progress was good in 11 patients $(91.67 \%)$ and regular in one patient $(8.33 \%)$. Conclusion: It is the first series communicated in Ecuador, where reference is made to the use of tibial osteotomy in the supramaleolar area, combined with Kirschner pin fixation, correcting internal tibial torsion in children where conservative treatment failed, resulting in a safe procedure and favorable results in the evolution of gait, including patients with neuromuscular disease and under five years of age.

Keywords: Gait, treatment, tibial torsion, osteotomy, evolution.

\section{Nivel de evidencia: IV}

* Médico residente de segundo año del Postgrado de Cirugía Pediátrica.

* Médico residente de primer año del Servicio de Ortopedia y Traumatología.

$\S$ Médico Especialista en Ortopedia y Traumatología.

Hospital de Niños «Dr. Roberto Gilbert Elizalde», Guayaquil, Ecuador.

Correspondencia:

Jorge Alejandro Oliveros-Rivero

E-mail: jorgealejandrooliveros@hotmail.com

Citar como: Oliveros-Rivero JA, Mendoza-Saldarreaga LG, Forestieri-Flores M, Quiñonez-León J, Melgar-Celleri J. Manejo quirúrgico de la marcha por torsión tibial interna en niños. Acta Ortop Mex. 2020; 34(5): 267-271. https://dx.doi.org/10.35366/97986 


\section{Introducción}

El primero en describir la torsión tibial fue Le Damany en 1903: «La torsión de la tibia alrededor de su eje longitudinal, resulta en un cambio en la alineación de los planos de movimiento de la articulación proximal (rodilla) y (tobillo) articulación distal». ${ }^{1}$ Cuando la medición de estas torsiones es superior a dos desviaciones estándar (DE) respecto a la edad e incluso al sexo, se habla de anomalías rotacionales de los miembros inferiores. ${ }^{2}$

La marcha con rotación interna puede ser consecuencia de trastornos anatómicos estáticos como metatarso aducto, torsión tibial interna y anteversión femoral aumentada; o desviaciones dinámicas de la marcha del plano transversal a nivel del pie, la rodilla, la cadera y la pelvis. ${ }^{3}$ La rotación interna más extrema, debido al aumento de la torsión tibial interna, puede alterar la función de la marcha al comprometer la fase de balanceo, también puede comprometer la estética de la marcha y ésta es una gran preocupación para padres e hijos, particularmente durante los años de escuela básica. ${ }^{3} \mathrm{El}$ diagnóstico de la torsión tibial interna se puede realizar mediante la historia clínica y el examen físico, se recomienda evaluar el ángulo muslo-pie (AMP), el cual se realiza con el niño en decúbito prono y las rodillas flexionadas a $90^{\circ}$, el AMP es la diferencia angular entre los ejes del pie y del muslo; ${ }^{3}$ la gravedad se obtiene por el ángulo muslo-pie (AMP) menor de $-15^{\circ}$ grave, menor de $-10^{\circ}$ moderado, menor de $-5^{\circ}$ leve, normal de $10 \mathrm{a} 15^{\circ}$. También se recomienda valorar la progresión del ángulo del pie (PAP) cuando el niño camina, ésta es la diferencia angular entre el eje del pie y la línea de progresión. Se determina el grado medio de marcha en abducción o aducción de las puntas de los pies, se asigna un valor negativo al modo de caminar con las puntas de los pies torcidas hacia dentro, siendo de -5 a $-10^{\circ}$ leve, de -10 a $-15^{\circ}$ moderado, más de $-15^{\circ}$ es grave y normal es $10^{\circ} ., 5$

La osteotomía desrotadora de tibia es eficaz para corregir la rotación interna de la tibia. Está indicada sólo en los niños mayores de ocho años, aunque otra literatura indica realizarla en mayores de cinco años que tienen una torsión tibial interna con dos desviaciones estándar por encima de la me-

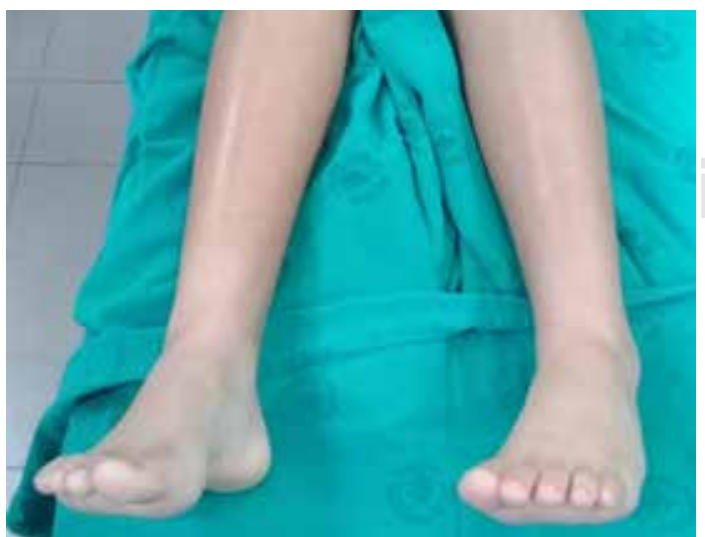

Figura 1: Paciente en decúbito supino, se operará tibia izquierda. dia. ${ }^{4,6} \mathrm{Si}$ la deformidad torsional necesita ser corregida, se requerirá una osteotomía de desrotación de la tibia. La osteotomía se puede realizar en la región supramaleolar, en la diáfisis o en la región metafisaria proximal. ${ }^{7}$ Una osteotomía en la región supramaleolar es relativamente fácil de realizar y es menos probable que se complique con una lesión de los vasos o un síndrome compartimental. Si hay una deformidad asociada en varo o valgo del tobillo, también se puede corregir a este nivel. La osteosíntesis con una pequeña placa recta angosta o la osteodesis con dos alambres cruzados de Kirschner es adecuada. Incluso si sólo un lado necesita ser corregido, ambas piernas deben estar libres. Esto permite que el cirujano se asegure de que se restablezca la simetría.

El propósito de este estudio fue identificar la evolución en la marcha de los pacientes que presentaron el diagnóstico de torsión tibial interna y se les trató con corrección quirúrgica realizándoles osteotomía tibial desrotadora.

\section{Material y métodos}

Se llevó a cabo un estudio retrospectivo, en el que se revisaron las historias clínicas de los pacientes a los que se les realizó osteotomía de tibia desrotadora por presentar el
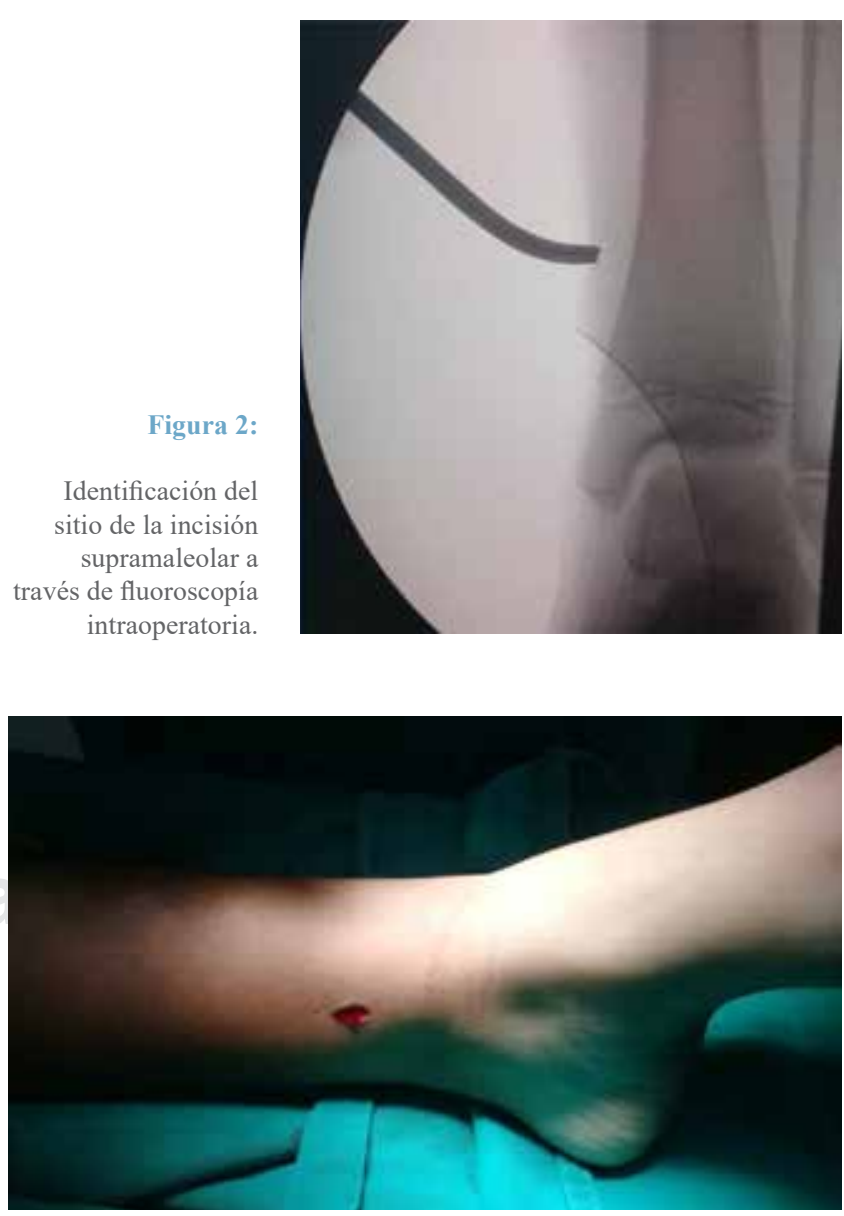

Figura 3: Incisión longitudinal en región supramaleolar de miembro inferior izquierdo. 

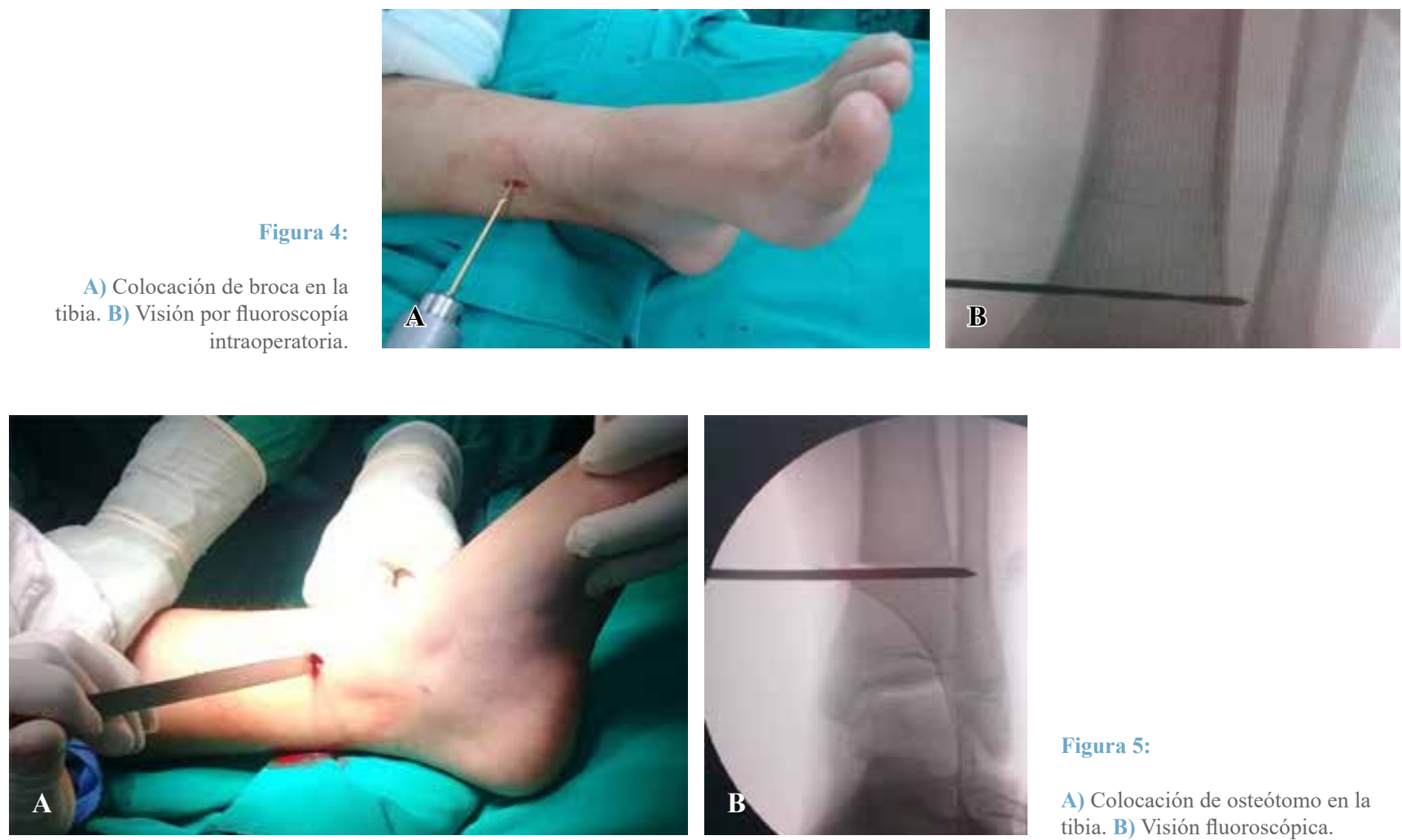

Figura 5:

A) Colocación de osteótomo en la tibia. B) Visión fluoroscópica.

diagnóstico de alteración de la marcha por torsión tibial interna, entre Enero de 2016 y Enero de 2019. Se incluyeron a todos los pacientes atendidos en el servicio con el diagnóstico de torsión tibial interna causante de una marcha anormal en quienes fracasó el tratamiento conservador. Se determinó la edad, sexo, estado nutricional, patologías asociadas, tipos de procedimientos quirúrgicos, estancia hospitalaria. Las complicaciones se clasificaron como: mayor si requerían cirugía adicional, si el resultado final causaba deterioro o retraso importante en la marcha; se clasificaron como leve si se resolvían rápidamente con tratamiento local o con terapia física, que no afectara el resultado final y que no causara retraso en la marcha y en la evolución postoperatoria; se evaluó durante seis meses, a través de la marcha, la cual se clasificó en buena aquella en que los resultados clínicos mejoraron significativamente y el ángulo de progresión del pie se encontraba entre cinco a 10 grados, regular aquella en que los resultados clínicos mejoraron significativamente y el ángulo de progresión del pie se encontraba entre cinco a cero grados y mala si no existió mejoría clínica y el ángulo de progresión del pie se encontraba menor a cero grados. Los resultados se expresan en números enteros, promedios y porcentajes.

La técnica quirúrgica elegida fue osteotomía supramaleolar de tibia: con el paciente en decúbito supino sobre la mesa de operaciones (Figura 1). Se identificó el sitio de la incisión supramaleolar a través de fluoroscopía (Figura 2), la tibia distal se expone a través de una incisión vertical de $1 \mathrm{~cm}$ a nivel supramaleolar (Figura 3 ). Se identifica la fisis distal de la tibia mediante fluoroscopía intraoperatoria para evitar el daño en ella. La osteotomía se hizo con broca en forma de abanico (Figura 4 A y B) y un osteótomo (Figura $5 A$ y $B$ ). Se hace la corrección de la rotación tibial y se fija con un clavo tipo Kirschner (el clavo debe estar bloqueado con el yeso), la posición final se verifica por fluoroscopía. La herida se cierra y la pierna es inmovilizada con un yeso debajo de la rodilla. Cuatro a seis semanas después de la operación se cambia el yeso y se retiran los clavos de Kirschner; se toman radiografías y se aplica un nuevo yeso; este segundo yeso se retira ocho semanas después de la operación, se toman nuevas radiografías y se observa la formación de callos. El paciente debe ser monitoreado cuidadosamente para detectar signos de falta de unión, como dolor o fractura de la osteotomía y la evolución de la marcha.

\section{Resultados}

Durante el período del estudio se les realizó osteotomía desrotadora de tibia a 12 pacientes, nueve del sexo femenino $(75 \%)$ y tres del sexo masculino $(25 \%)$. El mayor número de pacientes a los que se les realizó osteotomía tibial se encuentran en el grupo de 10 o más años de edad en quienes se efectuaron seis osteotomías (50\%) (Figura 6); el rango de edad al momento de la cirugía fue desde 4.3 hasta 17.8 años, con mediana de edad en 9.39 años. Se realizó la cirugía en dos pacientes que tenían menos de cinco años debido a que ambos presentaban un grado de rotación tibial interna severa y presentaban como patología asociada mielomeningocele y parálisis cerebral infantil. Del total de los pacientes a los que se les realizó osteotomía tibial se encontraron ocho 
pacientes $(66.67 \%)$ con un estado nutricional normal (Figu$\mathrm{ra} \mathrm{7).} \mathrm{El} \mathrm{rango} \mathrm{de} \mathrm{peso} \mathrm{al} \mathrm{momento} \mathrm{de} \mathrm{la} \mathrm{cirugía} \mathrm{fue} \mathrm{desde}$ 12.36 kilogramos hasta 59.30 kilogramos, con mediana de peso en 31.47 kilogramos.

De todos los pacientes operados tres tenían mielomeningocele $(25 \%)$, dos algún tipo de parálisis cerebral infantil como patología asociada (16.6\%), cuatro otras patologías asociadas y tres no tenían ninguna patología $(25 \%)$, por lo que se les consideró como torsión tibial pura.

Los datos de la estancia hospitalaria se resumen en la $\mathrm{Fi}$ gura 8. Se consideraron cuatro complicaciones, tres de ellas menores $(25 \%)$ representadas por atrofia muscular del tríceps sural, la cual se corrigió con terapia física con evolución satisfactoria y una de ellas mayores $(8.33 \%)$ representada por fractura del sitio de la osteotomía por inicio de la marcha antes de la autorización médica, la cual requirió una reintervención. En la actualidad, la paciente permanece en terapia física. Por último, ocho pacientes $(66.67 \%)$ no presentaron ninguna complicación. Los 12 pacientes incluidos en el estudio al realizarles el examen físico preoperatorio presentaron una progresión del ángulo del pie de menor a $-10^{\circ}$. Al realizar la evaluación de la marcha posterior a la cirugía correctora se evidenció una buena evolución en 11 pacientes (91.67\%) y sólo un paciente (8.33\%) presentó una marcha regular, ya que persistió marcha agazapada en menor intensidad con leve dolor.

\section{Discusión}

La osteotomía tibial proximal conlleva un alto riesgo de parálisis del nervio peroneo y el síndrome compartimental y debe reservarse para la corrección de genu varum o genu valgum. Estudios previos informaron una tasa de complicaciones mayores de $13 \%$ después de la osteotomía tibial proximal, incluida una tasa de parálisis del nervio peroneo de $5 \%,{ }^{8,9}$ estas complicaciones superan las encontradas en nuestro estudio que fue de sólo $8.33 \%$, acotando que la complicación mayor que tuvo fue porque el paciente no siguió las indicaciones médicas.

En la literatura revisada se hace mención a la ventaja que se tiene cuando la fijación se realiza con clavos de Kirsch-

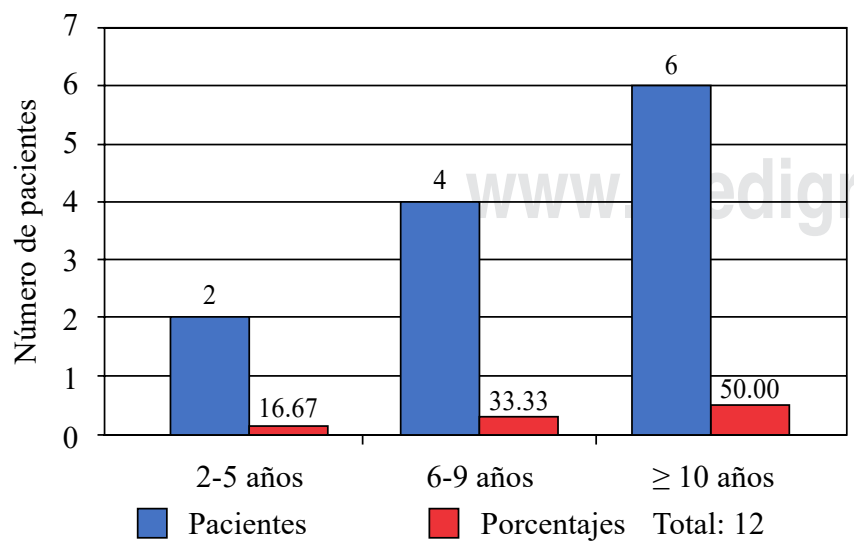

Figura 6: Distribución de pacientes por edad. ner en el sitio de la osteotomía, ya que realizar la fijación con placa tiene como desventaja someter al paciente a otra intervención para realizar el retiro de la placa. ${ }^{9}$ Después de observar los resultados del estudio, se sigue recomendando la fijación de la osteotomía con clavos de Kirschner en niños, ya que su retiro se puede realizar en la consulta.

Al observar el estudio realizado por Dodgin y colaboradores,$^{10}$ comparándolos con el resto de la literatura, no es concluyente sobre la necesidad de una osteotomía del peroné, además también se describe en la literatura que la osteotomía de peroné asociada a osteotomía de tibia presenta mayores probabilidades de producir alteraciones de tipo recurvatum o antecurvatum, lo cual causa mayor inestabilidad de la marcha. En esta serie, la osteotomía de peroné no se llevó a cabo en ninguno de los 12 pacientes a los que se les realizó la osteotomía desrotadora de tibia.

Es importante resaltar que tres pacientes presentaron mielomeningocele y dos pacientes parálisis cerebral infantil como patología primaria. La causa de que estos pacientes presenten torsión tibial interna es probablemente debido al entorno biomecánico anormal durante el crecimiento; ${ }^{9}$ pero aun así, los pacientes tuvieron una evolución satisfactoria con respecto a la marcha, similar a los pacientes que no tenían ninguna patología asociada; por lo tanto, esta corrección les puede ayudar a mejorar la biomecánica de su marcha.

La mediana de edad fue de 9.39 años, cumpliendo lo recomendado por la literatura mundial ${ }^{6}$ sin embargo, se realizó la cirugía en dos pacientes que tenían menos de cinco años debido a que ambos presentaban un grado de rotación tibial interna severa y presentaban como patología asociada mielomeningocele y parálisis cerebral infantil; en ambos casos se obtuvieron evoluciones satisfactorias en la marcha y no presentaron complicaciones mayores ni menores en el postoperatorio.

Múltiples estudios previos han sugerido que el sitio óptimo para una osteotomía desrotadora por torsión tibial interna pura es el área supramaleolar de la tibia distal, ${ }^{9}$ además de esto se puede observar que en los pacientes que tenían patologías asociadas, y en especial las neurológicas, también fueron beneficiados con este tipo de tratamiento. Es

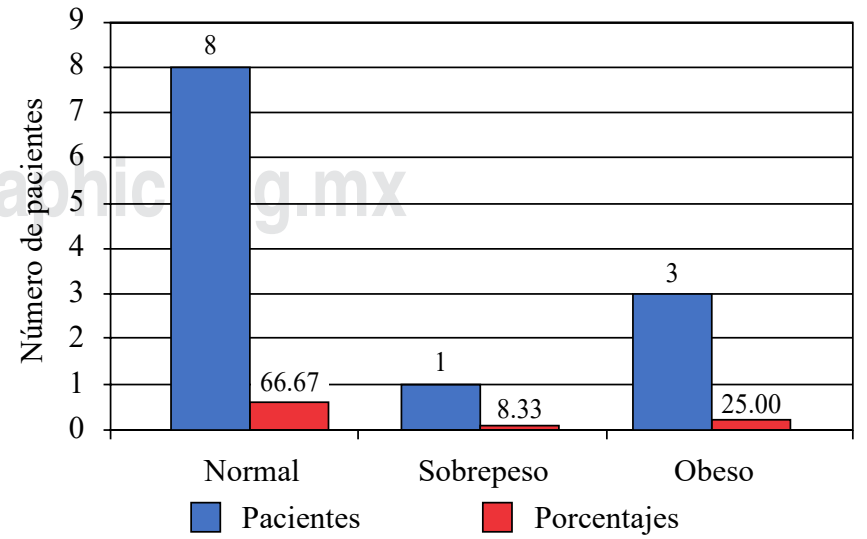

Figura 7: Distribución de pacientes por estado nutricional. 


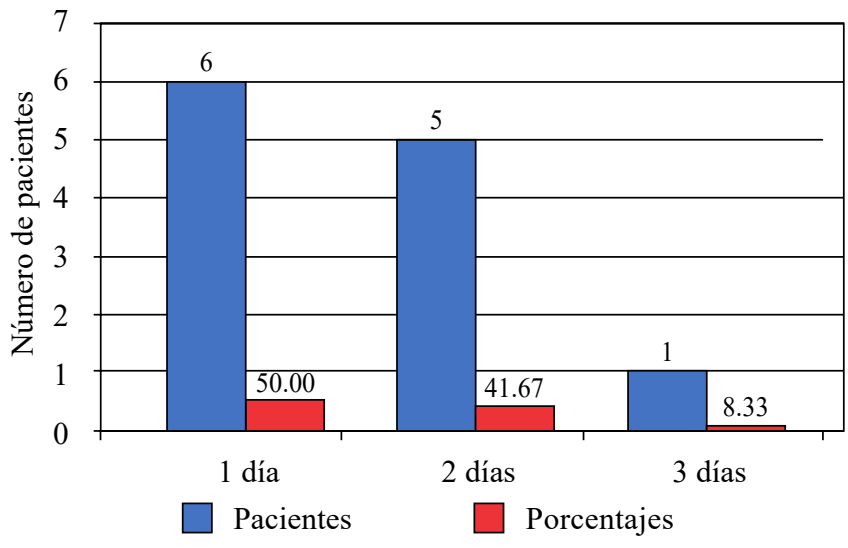

Figura 8: Distribución de estancia hospitalaria postoperatoria.

importante mencionar que a los 12 pacientes incluidos en el estudio, al realizarles el examen físico preoperatorio, presentaron una progresión del ángulo del pie de menor a $-10^{\circ}$. $\mathrm{Al}$ evaluar la marcha posterior a la cirugía correctora se evidenció una buena evolución en 11 pacientes (91.67\%), los cuales presentaron una progresión del ángulo del pie mayor a ocho grados, con mejoría funcional y estético; sólo un paciente $(8.33 \%)$ presentó una marcha regular, ya que persistió marcha agazapada en menor intensidad con leve dolor y una progresión del ángulo del pie de menor a cinco grados pero mayor a cero grados.

Nuestras principales limitantes son la pequeña muestra y lo variado de las etiologías de la torsión tibial; carecemos de un grupo control que confirme la validez de resultados y, por supuesto, nuestro seguimiento a tan corto plazo no nos permite evaluar el impacto de la osteotomía en el crecimiento ni en la deformación plástica del peroné.

\section{Conclusión}

Es la primera serie comunicada en Ecuador de este tratamiento; en el corto plazo consideramos un procedimiento eficaz, con buena consolidación del área de la osteotomía, pocas complicaciones, incluidos pacientes menores de cinco años.

\section{Referencias}

1. Pons FA. Importancia de las torsiones tibiales en el desarrollo infantil de los 4 a 7 años. [Trabajo final de grado]. Barcelona: Universitat de Barcelona; 2015, 48.

2. Accadbled F, Mansat C, Cahuzac J-P. Anomalies rotationnelles des membres inférieurs chez l'enfant. Paris: EMC (Elsevier Masson SAS, Paris); 2007.

3. Davids JR, Davis BR, Jameson LC, David EW, James WH. Surgical management of persistent intoeing gait due to increased internal tibial torsion in children. J Pediatr Orthop. 2014; 34(4): 467-73.

4. Staheli LT. Ortopedia pediátrica. Madrid: Marbán; 2003.

5. Chauca JC. Deformidades torsionales de los miembros inferiores y la alteración del equilibrio dinámico en niños de 4 a 7 años. [Trabajo final de grado]. Lima: Universidad Nacional Mayor de San Marcos; 2008. 76 .

6. Losada BP. Alteraciones de los miembros inferiores: deformidades angulares, torsionales, alteraciones de la marcha y dismetrías. Pediatr Integral. 2002; 6(5): 397-412.

7. Joseph B, Nayagan S, Loder R, Torode I. Paediatric orthopaedics. 2nd ed. Florida: CRC press; 2016.

8. Krengel WF, Staheli LT. Tibial rotational osteotomy for idiopathic torsion: a comparison of the proximal and distal osteotomy levels. Clin Orthop Relat Res. 1992; 283: 285-9.

9. Selber P, Filho ER, Dallalana R, Pirpiris M, Nattrass GR, Graham HK. Supramalleolar derotation osteotomy of the tibia, with T plate fixation. J Bone Joint Surg. 2004; 86(B): 1170-5.

10. Dodgin DA, De Swart RJ, Stefko RM, Wenger DR, Ko J. Distal tibial/fibular derotation osteotomy for correction of tibial torsion: review of technique and results in 63 cases. J Pediatr Orthop. 1998; 95-101 